\title{
Clinical trials in rheumatology: an uncertain future?
}

\author{
Alan J Silman
}

In evidence-based medical practice, therapeutic decisions should be guided by robust scientific evidence rather than clinical opinion or prejudice. Such evidence is ideally based on meta-analyses of several well-conducted randomized controlled clinical trials, although if necessary, a single trial can suffice. The use of non-evidence-based therapeutic decisions is increasingly subject to scrutiny from health care providers and professional bodies, but does evidence-based practice constrain clinical freedom or ensure optimal standards of care?

The perception of the efficacy or tolerability of treatments that an individual physician gains from clinical experience does not always reflect the results found in clinical trials. It is appropriate to question why there is a disparity between clinical trials and clinical experience. The simplistic question to ask is whether it is the trial or the perception gained from clinical experience that is flawed.

Perhaps the greatest problem with clinical trials in rheumatology is that we are frequently seeking modest differences in relatively modest therapeutic goals. For instance, a 20\% response in the American College of Rheumatology criteria (ACR20) is seen as a positive result in a clinical trial, but it is unlikely to be seen by either patient or physician as a success. As a consequence, the clinical trial data have little influence on treatment decision making.

Another problem is that there are an infinite number of therapeutic questions in any given clinical situation, and only a fraction of these questions are subject to scrutiny in trials. For example, most trials of co-therapy with a disease-modifying antirheumatic drug and an anti-tumor-necrosis-factor (anti-TNF) agent for rheumatoid arthritis have involved the use of methotrexate. An important proportion of patients is, however, intolerant to methotrexate, so co-therapy with another disease-modifying antirheumatic drug, such as sulphasalazine or leflunomide, might be appropriate-there are
It is

appropriate

to question

why there is

a disparity

between

clinical trials

and clinical

experience

AJ Silman is an Advisory Board member of Nature Clinical Practice Rheumatology.

\section{Competing interests}

The author declared he has no competing interests.

www.nature.com/clinicalpractice doi:10.1038/ncprheum0164 more limited trial data that address these questions. No data on efficacy is not the same as data showing no efficacy; so in this regard, the available data from trials are inadequate.

The choice of questions that are asked in clinical trials is frequently dictated by regulators (especially in the US) and by pharmaceutical companies, and their requirements might not reflect the therapeutic demands of routine clinical care. Some questions, such as which is the best first choice out of the three currently available anti-TNF agents for rheumatoid arthritis, will remain unanswered by direct comparison between the available agents. Investigator-initiated clinical trials that could address these unanswered questions are infrequent, for obvious reasons related to the cost of undertaking a sufficiently large study and to the increasing complexity surrounding the ethics and governance of clinical trials.

In many trials, there are an important number of individuals in both arms who do not respond to treatment. A trial cannot address whether it would be the same individuals in each of the two arms who would not respond. As an example, it seems likely that anti-TNF therapy is better than methotrexate for the early treatment of rheumatoid arthritis, though the latter is successful in a substantial proportion of patients. The important questions are whether methotrexate responders can be robustly identified, and in those who do not respond to methotrexate, whether TNF responders can be similarly identified.

The aim for the future is that therapeutic decisions will be based on the characteristics of the individual. Clinical and currently available laboratory predictors are too crude to be of use, but pharmacogenetic and metabolomic profiling might permit this in the future. The studies that will identify these predictors are carefully conducted observational studies in real-world patients. The 'political' question is how such answers will rank in the hierarchy of evidence. 